\title{
Impact of CMS measurements on PDFs
}

\author{
Nicole Ruckstuhl ${ }^{1, a}$ \\ ${ }^{1}$ University of Minnesota, School of Physics and Astronomy, 116 Church Street S.E., Minneapolis MN55455
}

\begin{abstract}
.
Recently 2 analyses have been published by the CMS collaboration that study the effect of a specific CMS measurement on PDFs. A review of these studies is given here.

The first analysis consists of a double differential inclusive jet cross-section measurement, performed on $4.7 \mathrm{fb}^{-1}$ of data at $\sqrt{\mathrm{s}}=7 \mathrm{TeV}$. Using the HERAFitTer code, the effect of adding this measurement in the PDF fit procedure together with DIS data from HERA-I is studied. The CMS data is able to significantly reduce the uncertainties on the gluon PDF, especially for gluons with large momenta relative to the proton $(x>0.01)$. A moderate reduction in the uncertainty of the $\mathrm{u}$ and $\mathrm{d}$ valence quark PDFs is also observed for $x>0.01$.

The quark PDFs are more directly probed by gauge boson production. Therefore the second analysis studies the effect of W-boson production measurements on PFDs. This consists of a W-boson charge asymmetry measurement and a study of W-boson production in association with a c-(anti-)quark, both performed on $5.0 \mathrm{fb}^{-1}$ of data at $\sqrt{\mathrm{s}}=7 \mathrm{TeV}$. A clear reduction in the uncertainty on the $\mathrm{u}$ and $\mathrm{d}$ valence quark PDFs is seen for $x<0.1$. Furthermore the W-boson + c-quark production measurements allow for the determination of an independent $\mathrm{s}$ quark PDF, which is not possible on DIS data alone.
\end{abstract}

\section{Introduction}

Any process occurring in a proton-proton collision is naturally dependent on the composition of partons within the protons. For the purpose of making theoretical predictions of these processes, the proton composition is modeled in parton distribution functions, PDFs, which give the parton content as a function of the momentum fraction, $x$, of the proton carried by the parton. The PDFs depend on the energy scale, $Q$, considered, with the evolution from one energy scale to another being fully described by perturbative QCD. The PDFs at a start-up energy scale $Q_{0}$ include non-perturbative effects and need to be modeled and fitted to a wide range of experimental data. Many PDF sets are currently in use, differing in parametrization and the data used to fit them [1-5].

Uncertainties on PDFs are an important consideration for interpreting any analysis at a hadron collider such as the LHC. Reversely, specific measurements at the LHC, where other uncertainties are relatively small, can be used to help test and improve our understanding of PDFs. Since the start up of the LHC, the CMS detector has collected an integrated luminosity of approximately $5 \mathrm{fb}^{-1}$ of pp collision data at a center of mass energy of $7 \mathrm{TeV}$ and $20 \mathrm{fb}^{-1}$ of pp data at $8 \mathrm{TeV}$. A wide range of measurements performed on this data could be and is already used by different PDF groups to improve their fits. The main impact comes from analyses falling within one of two categories: a measurement of jet production, which gives a good han-

\footnotetext{
a e-mail: nicole.manuela.ruckstuhl@cern.ch
}

dle on the gluon content of protons, especially for large $x$, or of gauge boson production which is more sensitive to the quark content.

The aim of this article is to discuss the effect of CMS measurements on PDFs, by focusing on three analyses for which the CMS collaboration has studied the direct effect on PDFs using the HERAFITTER framework [4, 6, 7]. These are: an inclusive multi-jet differential cross-section measurement [8, 9], a W-boson charge asymmetry measurement in events where the $\mathrm{W}$-boson decays to a muon and a neutrino [10] and a study of the W-boson production in association with a c-quark [11].

The remainder of this article is structured as follows. Section 2 gives a short introduction to HERAFITTER. It is followed by a short overview of the CMS detector and the data samples involved. Section 4 describes the inclusive jet analysis and its impact on PDFs while section 5 does the same for the two W-boson analyses. The last section provides an overall conclusion and summary.

\section{HERAFITTER framework}

For most of the relevant range in the $(x, Q)$ plane, the tightest constrains on the PDFs is provided by deep inelastic lepton-proton scattering (DIS) data from HERA-I [4]. It is mostly at higher values of $x$ where data from the LHC becomes valuable. The effects on PDFs from the analyses described in this paper are therefore studied by comparing PDFs fitted solely on DIS data from HERA with PDFs fitted on the combination of this data with the CMS results. 
This is done using the HERAFITTER code, an open source framework using the QCDNUM package [12] to evolve the partons between energy scales. The calculations as well as the HERA data used, follow the prescription for HERAPDF.

In the default setting the HERAFitTER fits five independent combinations of parton distributions using the following parameterization:

$$
\begin{aligned}
x g(x) & =A_{g} x^{B_{g}}(1-x)^{C_{g}}-A_{g}^{\prime} x^{B_{g}^{\prime}}(1-x)^{C_{g}^{\prime}}, \\
x u_{v}(x) & =A_{u_{v}} x^{B_{u_{v}}}(1-x)^{C_{u_{v}}}\left(1+E_{u_{v}} x^{2}\right), \\
x d_{v}(x) & =A_{d_{v}} x^{B_{d_{v}}}(1-x)^{C_{d_{v}}}, \\
x \bar{U}(x) & =A_{\bar{U}} x^{B_{\bar{U}}}(1-x)^{C_{\bar{U}}}, \\
x \bar{D}(x) & =A_{\bar{D}} x^{B_{\bar{D}}}(1-x)^{C_{\bar{D}}},
\end{aligned}
$$

describing, in order, the gluon PDF, the $u$ and the $d$ valence quark PDFs, the $u$ sea quark PDF and finally the combined $d$ and s sea quark PDF. By default the PDF for $\mathrm{s}$-quarks is taken to be proportional to that for the $\mathrm{d}$ sea quark with a proportionality factor of $f_{s}=\bar{s} /(\bar{d} \bar{s})=0.31 \pm$ 0.08 [13].

The parameters $A_{g}, A_{u_{v}}$ and $A_{d_{v}}$ of equation 1 are fixed by QCD sum rules. Furthermore, $B_{\bar{U}}=B_{\bar{D}}$ and $A_{\bar{U}}=$ $A_{\bar{D}}\left(1-f_{s}\right)$ are set to ensure equal $\bar{u}$ and $\bar{d}$ densities at $x \rightarrow$ 0 . Finally $C_{g}^{\prime}$ is set to $25[2,14]$. This leaves 13 free parameters to be fitted to the data.

Three categories of uncertainties on these PDFs are determined. The experimental uncertainty is the propagated uncertainty from the experimental data, taking into account the documented correlations between these measurements. The parametrization uncertainty is calculated by using more flexible functions for the different PDFs, such as $A x^{B}(1-x)^{C}(1+D x)$ or $A x^{B}(1-x)^{C}\left(1+D x+E x^{2}\right)$. Finally the model uncertainty is calculated by varying fixed parameters of the model within their given uncertainty. This includes varying the b-quark mass from its default value of $4.75 \mathrm{GeV}$ in the range 4.3 to $5 \mathrm{GeV}$, varying the c-quark mass from $1.4 \mathrm{GeV}$ in the range between 1.3 and $1.65 \mathrm{GeV}$ and changing the range of the HERA DIS data included.

More details on the precise procedure and the settings used can be found in Ref. [9, 10].

\section{CMS detector}

The analyses described in this paper are performed on data collected by the CMS detector in 2011, corresponding to to an integrated luminosity of 4.7 and $5.0 \mathrm{fb}^{-1}$ of protonproton collision data at a center of mass energy of $7 \mathrm{TeV}$ for the inclusive jet cross-section and the W-boson measurements respectively.

The central feature of the CMS detector is a superconducting solenoid of $6 \mathrm{~m}$ internal diameter, providing a magnetic field of $3.8 \mathrm{~T}$. Within the superconducting solenoid volume are a silicon pixel and strip tracker, surrounded by a lead tungstate crystal electromagnetic calorimeter and a brass/scintillator hadron calorimeter. Outside the field volume, in the forward region a iron/quartz-fiber hadronic calorimeter increases the CMS coverage. Muons are measured in gas-ionization detectors embedded in the steel flux-return yoke outside the solenoid.

The CMS coordinate-system has its origin at the nominal interaction point in the center of the detector. It is a right-handed coordinate-system, with the $z$ axis pointing along the counterclockwise beam direction and the $\mathrm{x}$-axis to the center of the LHC ring. The azimuthal angle is denoted by $\phi$, the polar angle by $\theta$ and the pseudo-rapidity is defined as $\eta=-\ln [\tan (\theta / 2)]$.

A more detailed description of the CMS apparatus can be found in Ref. [15].

\section{Inclusive jet differential cross-section measurement}

Jet production dominates over any other process at the LHC and is directly dependent on the PDFs. Very high energetic jets are a clean way to study the gluon PDF at high $x$. This section summarizes an inclusive jet doubledifferential cross-section measurement to the transverse momentum, $p_{\mathrm{T}}$, and absolute rapidity, $|y|$, of the jet. For more details of this analysis please see the original paper [8].

\subsection{Measurement}

Events for this analysis were selected using a range of single jet triggers with different $p_{\mathrm{T}}$ thresholds. All but the most stringent of the selected trigger (with $p_{\mathrm{T}}>370 \mathrm{GeV}$ ) were prescaled. Offline, the jets are reconstructed using the anti- $k_{T}$ clustering algorithm [16] with distance parameter $R=0.7$ as included in the FASTJET package [17].

The double-differential cross-section is then given by:

$$
\frac{\mathrm{d}^{2} \sigma}{\mathrm{d} p_{\mathrm{T}} \mathrm{d} y}=\frac{1}{\epsilon \cdot \mathcal{L}_{\text {int }}} \frac{N_{\text {jets }}}{\Delta p_{\mathrm{T}}(2 \cdot \Delta|y|)},
$$

where $N_{\text {jets }}$ denotes the number of reconstructed jets in the considered bin, $\epsilon$ is the combination of event reconstruction and trigger efficiencies, $\mathcal{L}_{\text {int }}$ is the integrated luminosity and $\Delta p_{\mathrm{T}}$ and $\Delta|y|$ denote the width of the bin.

The measured differential cross-section for five rapidity bins up to $|y|=2.5$ and for jet $p_{\mathrm{T}}$ up to $2 \mathrm{TeV}$ is shown in figure 1. The data is compared to theoretical predictions based on a NLO QCD calculation of the jet cross-section performed with the NLO-Jet++ program (v2.0.1) [18] within the fastNLO package (v1.4) [19]. Non-perturbative corrections are applied to account for multi parton interactions (MPI) and hadronization effects. The renomalization and factorizations scales are fixed to the jet $p_{\mathrm{T}}$. As defaults the PDFs from NNPDF2.1 [20] were used.

There is good agreement between the data and the theoretical prediction over the entire kinematic range. For a better comparison figure 2 shows the ratio between the experimental measurements and the theoretical prediction for the most central (top) and most forward (bottom) rapidity bins. The ratios of the theoretical predictions obtained 


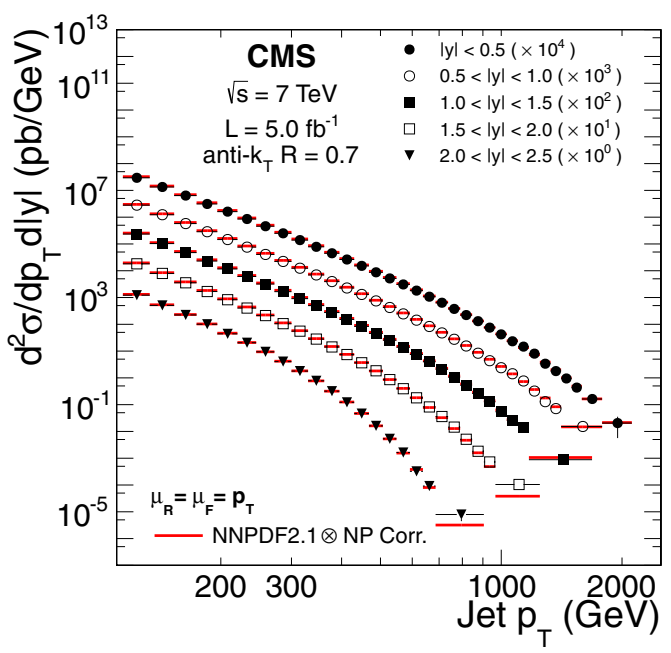

Figure 1. Inclusive jet cross-section for data (marker) and theory using the NNPDF2.1 PDF set (thick line) for different rapidity bins [8].
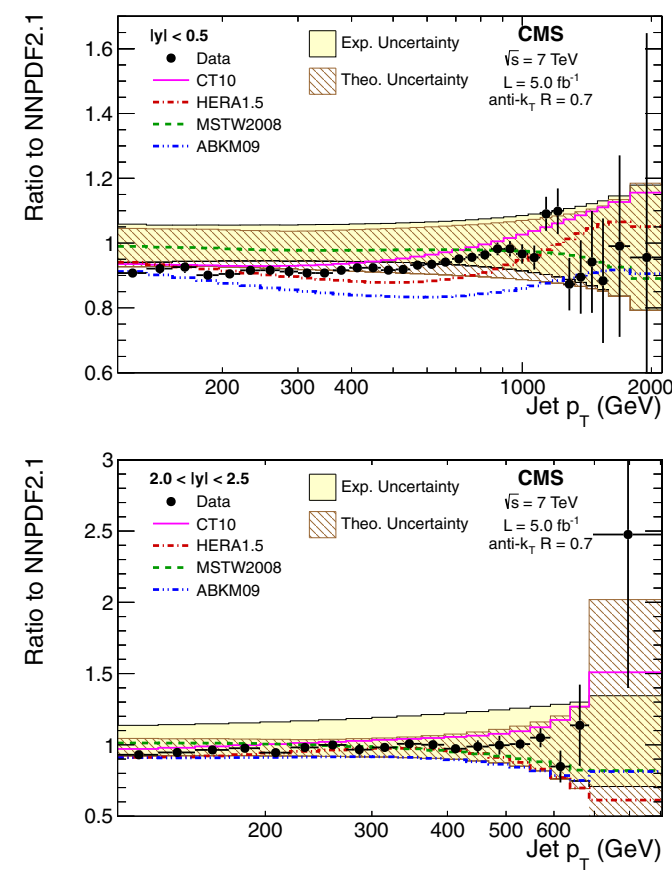

Figure 2. Ratio of the inclusive jet cross-section over the theoretical prediction using the NNPDF2.1 PDF set (black dots) for jets in the range $|y|<0.5$ (top) and $2.0<|y|<2.5$ (bottom) [8]. The continuous (hatched) bands show the total experimental (theoretical) uncertainty while the pink line and the dotted/dashed lines show the ratio for the cross-sections calculated using other PDF sets to the NNPDF2.1 predictions.

with other PDF sets, CT10 [1], MSTW2008NLO [2], HERAPDF1.5 [4] and ABKM09 [5], compared to the results obtained with NNPDF2.1 are also shown.
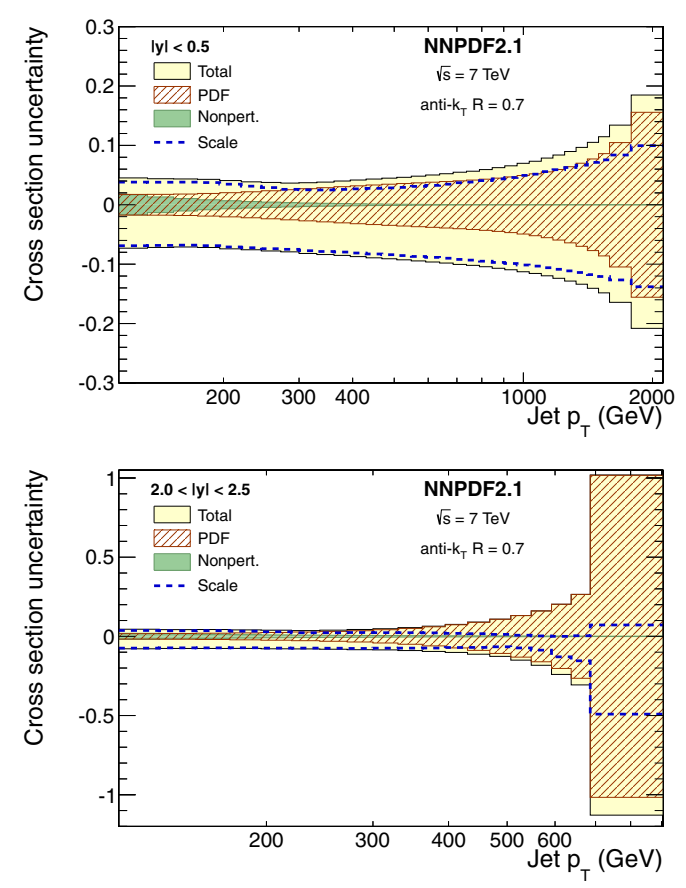

Figure 3. The relative theoretical uncertainties for the inclusive jet cross-section for jets in in the range $|y|<0.5$ (top) and $2.0<$ $|y|<2.5$ (bottom) [8].

\subsection{Uncertainties}

The size of the total experimental and theoretical uncertainty is also indicated in figure 2. The experimental uncertainty is dominated over the entire range by the uncertainty on the jet energy scale. This scale represents any bias in the reconstructed energy of jets compared to the true energy of the particles. Due to the steeply falling distributions as a function of $p_{\mathrm{T}}$ a relative small uncertainty in the energy scale of 2-2.5\% leads to an uncertainty of 5$30 \%$ on the final distribution. 16 interdependent sources of uncertainties on the jet energy scale have been identified, including: pile-up effects, relative calibration of jet energy as a function of pseudo-rapidity, absolute energy scale as a function of $p_{\mathrm{T}}$ and differences between jets from quarks and gluons. The uncertainty from each source is considered fully correlated over the entire kinematic range, while they are considered fully uncorrelated between sources. A full description of these sources and the additional experimental uncertainties are given in Ref. [8].

The theoretical uncertainty is dominated at low $p_{\mathrm{T}}$ by the uncertainty on the choice in renomalization and factorizations scales obtained by varying them up and down by a factor of 2 . At high $p_{\mathrm{T}}$ the uncertainty on the PDFs starts to dominated. Figure 3 shows the contributions of the different theoretical uncertainties for the most central (top) and most forward (bottom) rapidity bins.

From this information it is clear that, especially for the high $p_{\mathrm{T}}$ range, the PDF uncertainty is one of the major uncertainties in comparing this experimental measurement to theoretical predictions, indicating that this analysis is very suitable to study PDFs. 


\subsection{Effect on PDFs}

The effect of this inclusive jet differential cross-section measurements on PDFs was studies in Ref. [9] and is summarized here.

Before including the data distribution of figure 1 in the HERAFITTER fit procedure, the measurement was corrected for non-perturbtive, NP, effects, specifically for the before mentioned MPI and hadronization effects. This was done by obtaining correction factors from the LO MC event generators PYTHIA6 [21] and HeRWIG++ [22] and the NLO MC event generator POWHEG [23-26] with showering, MPI and hadronization done by PYTHIA (See Ref [9] for specifics). The central value of the envelope of these three predictions in used as default value for the NP correction factor with the half spread as uncertainty.

The corrected data distributions with the full covariance matrix for the uncertainties was included with the HERA-I DIS data in the procedure described in section 2. The resulting gluon PDF is shown in the top plot of figure 4 together with the gluon PDF fitted only on the HERA-I DIS data. The CMS data introduces a clear reduction in the uncertainty, especially for $x>0.01$. Furthermore the CMS data seems to indicate a higher gluon concentration at high $x$ than the HERA data alone.

The $u$ and the $d$ valence quark PDFs fitted with and without the CMS jet data are shown in bottom two plots of figure 4. As expected the effect of the CMS data is smaller on these PDFs than on that of the gluon. Nevertheless a modest reduction in the size of the uncertainty is observed for $x>0.01$ values. The seemingly increased uncertainty at lower $x$ is an artifact of how the parametrization uncertainty is calculated and due to the very small contribution of valence quarks at these values of $x$, has a negligible absolute effect. There is no noticeable effect of the CMS jet data on the sea quark PDFs.

\section{W-boson production}

W-boson production at the LHC is dominated by the annihilation of a valence u- or d-quark with, respectively, a sea d- or u-anti-quark. Therefore W-boson studies can provide a handle multiple quark PDFs. When considering events where the W-boson was produced in combination with a single c-(anti-)quark the dominating processes are $s g \rightarrow W^{-}+c$ and $\bar{s} g \rightarrow W^{+} \bar{c}$. Such events give access to the s quark PDF.

This section describes a measurement of the W-boson charge asymmetry for $\mathrm{W}$-bosons decaying to muons and neutrinos [10] and a study of W-boson production in association with a c-quark [11] with the W-boson decaying to an electron or muon with a corresponding neutrino.

\subsection{W-boson charge asymmetry}

\subsubsection{Event selection}

Events were selected using a isolated single muon trigger with different $p_{\mathrm{T}}$ thresholds. The isolation is determined by considering both the transverse energy deposited in the
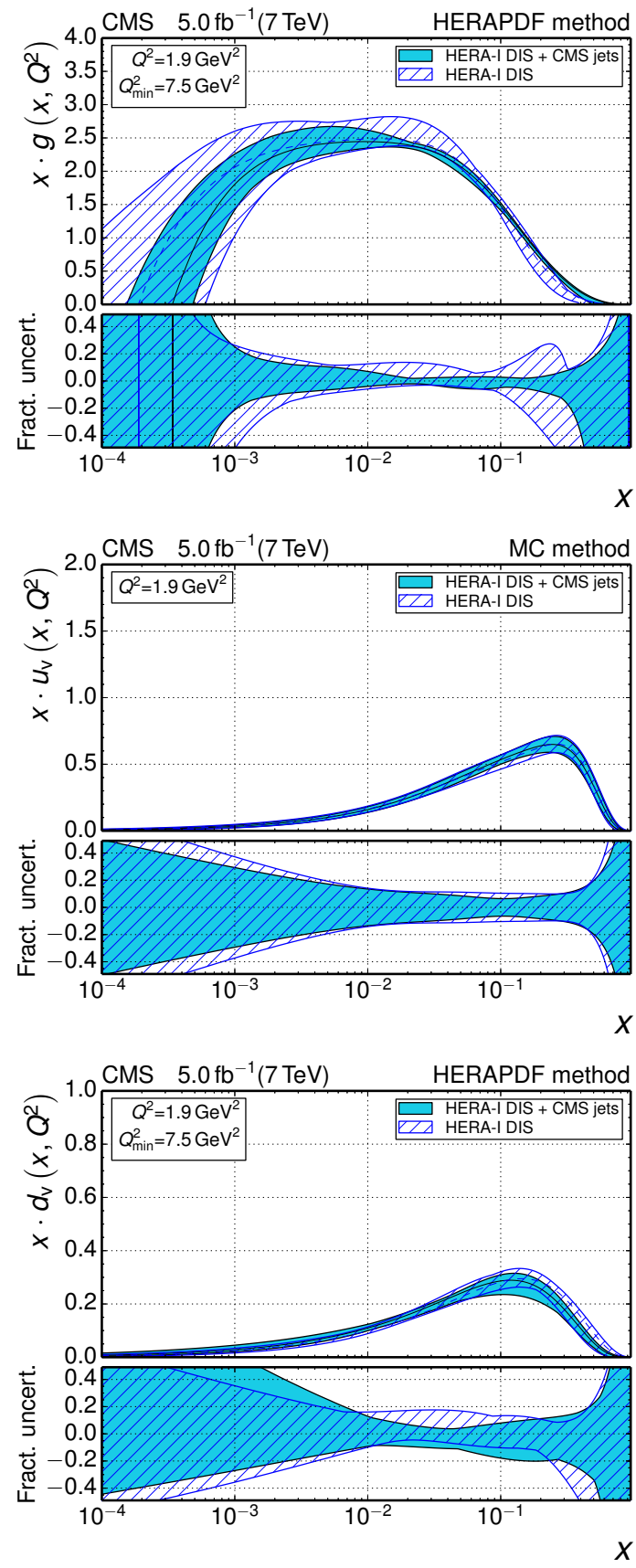

Figure 4. The gluon (top), u valence quark (middle) and d valence quark (bottom) PDFs fitted on the combination of HERA-I DIS data and CMS jet data (filled line) are shown together with the same PDFs fitted without the CMS data (dashed line) [9].

calorimeters and the sum of $p_{\mathrm{T}}$ of tracks in the area surrounding the muon candidate. Offline, the muon is required to have $p_{\mathrm{T}}>25 \mathrm{GeV},|\eta|<2.4$ and for the sum of additional tracks in the cone $\sqrt{(\Delta \eta)^{2}+(\Delta \phi)^{2}}<0.3$ to be less then $10 \%$ of the reconstructed muon $p_{\mathrm{T}}$. Here $\Delta \eta$ and $\Delta \phi$ denote the difference in $\eta$ and $\phi$ between the muon candidate and the considered track. Events with a second reconstructed muon with $p_{\mathrm{T}}>15$ are rejected to suppress the background from $Z / \gamma^{*} \rightarrow \mu^{+} \mu^{-}$. 
The neutrino produced in the decay of the W-boson can't be detected directly, but will produce a imbalance in the total transverse momentum of the event. The CMS experiment uses a particle-flow algorithm [27, 28] which, by the vectorial sum of the transverse momentum of all particles reconstructed by this algorithm, provides the missing transverse momentum of the event, denoted by $\mathscr{E}_{T}$. Instead of cutting on $\mathscr{E}_{T}$, the $\mathscr{E}_{T}$ distribution is used to fit the signal to background ratio of the selected events. The dominant backgrounds come from QCD multi-jet events and from Drell-Yan.

More information on the event selection, the fit procedure, and the different types of background can be found in Ref. [10].

\subsubsection{Charge asymmetry measurement}

The W-boson lepton charge asymmetry is measured as a function of the muon pseudo-rapidity and is given by:

$$
\mathcal{A}(\eta)=\frac{\frac{\mathrm{d} \sigma}{\mathrm{d} \eta}\left(W^{+} \rightarrow l^{+} v\right)-\frac{\mathrm{d} \sigma}{\mathrm{d} \eta}\left(W^{-} \rightarrow l^{-} \bar{v}\right)}{\frac{\mathrm{d} \sigma}{\mathrm{d} \eta}\left(W^{+} \rightarrow l^{+} v\right)-\frac{\mathrm{d} \sigma}{\mathrm{d} \eta}\left(W^{-} \rightarrow l^{-} \bar{v}\right)}
$$

The main advantage of measuring such a ratio, instead of the individual $W^{+}$and $W^{-}$cross-sections is that most experimental uncertainties cancel. Therefore the charge asymmetry in a $|\eta|$ bin is very close to

$$
\mathcal{A}^{\text {raw }}(\eta)=\frac{N^{W^{+}}-N^{W^{-}}}{N^{W^{+}}+N^{W^{-}}}
$$

where the number of $W^{+}\left(N^{W^{+}}\right)$and $W^{-}\left(N^{W^{-}}\right)$are obtained from the fit. Small corrections are applied to account for small differences in the $\mu^{+}$and $\mu^{-}$reconstruction and trigger efficiency due to detector misalignment.

The $|\eta|$-distribution of the $\mathrm{W}$-boson lepton charge asymmetry is shown in figure 5. A full description of the uncertainties is given in the original paper [10]. The dominant uncertainties are due to the remaining uncertainty in the difference in reconstruction and trigger efficiency between positively and negatively charged muons, and to the QCD background.

The measured lepton charge asymmetry is compared to theoretical predictions obtained with FEwz 3.1 [29] at NLO and interfaced with the CT10 [1], NNPDF2.3 [3, 31], HERAPDF1.5 [4], MSTW2008 [2] and MSTW2008CPdeut [32] PDF sets. From figure 5 we can see that CT10, NNPDF2.3 and HERAPDF1.5 are in good agreement with the data, but that predictions with MSTW2008 significantly underestimate the asymmetry especially at low $|\eta|$. The newer MSTW2008CPdeut, using a more flexible parametrization, provides better agreement.

From figure 5 it is clear that the total experimental uncertainty of this measurement is significantly smaller then the spread in theoretical predictions from the chosen PDFs. Therefore this analysis can be used to improve current existing PDF sets.

The same analysis was also preformed with a $p_{\mathrm{T}}$ threshold of $35 \mathrm{GeV}$. This has the advantage of greatly

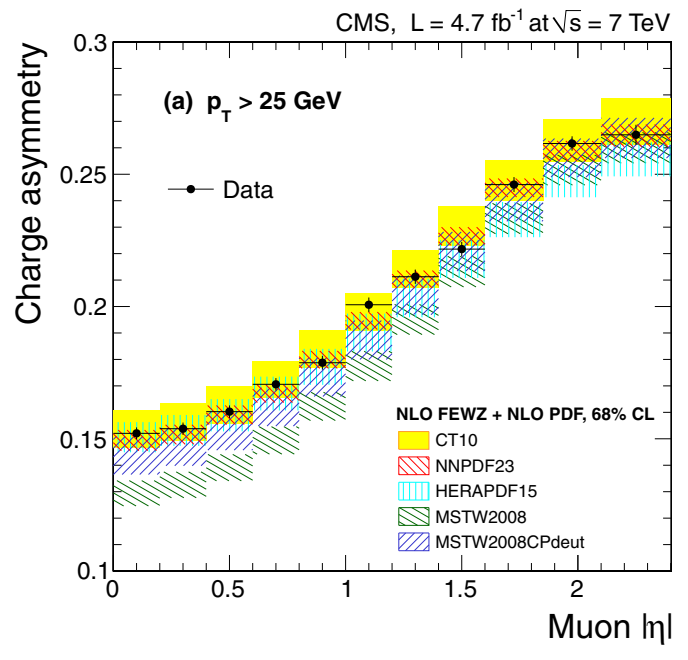

Figure 5. The W-boson muon charge asymmetry in data (black dots) compared to NLO predictions with different PDF sets [10].

reducing the QCD background. While the lepton charge asymmetry distribution changes with this new threshold, showing a smaller asymmetry, the conclusions with respect to the comparison with theoretical predictions are similar [10].

\subsection{W-boson + c-(anti-)quark production}

\subsubsection{Event selection}

This analysis considers W-boson decays in both the muon and the electron channel. Events were selected using either a single isolated muon trigger or an electron trigger. Offline, the reconstructed lepton was required to have $p_{\mathrm{T}}>35 \mathrm{GeV}$ and $|\eta|<2$.1. Furthermore electrons were excluded in the $1.44<|\eta|<1.57$ range. Electron isolation is enforced by requiring that the sum of the $p_{\mathrm{T}}$ of tracks in a cone of $\sqrt{(\Delta \eta)^{2}+(\Delta \phi)^{2}}=0.3$ around the electron candidate is less the $5 \%$ of the reconstructed electron $p_{\mathrm{T}}$. For the muon isolation a cone of size 0.4 is used and a relative threshold of $12 \%$.

Aside from lepton selections the transverse mass of the W-boson candidate, given by $M_{\mathrm{T}}=\sqrt{2 p_{\mathrm{T}}^{l} \mathscr{E}_{T}\left[1-\cos \left(\phi_{l}-\phi_{\not_{T}}\right)\right.}$, must be greater then 40 (50) $\mathrm{GeV}$ in the muon (electron) channel.

Events with a c-quark candidate are selected by requiring a jet reconstructed by the same anti- $k_{T}$ clustering algorithm as in section 4 but with a distance parameter of 0.5 , with $p_{\mathrm{T}}>25 \mathrm{GeV}$ and $|\eta|<2.5$. Jets from c-quarks are identified searching for specific decay channels. Three channels are considered: $\mathrm{D}^{ \pm} \rightarrow \mathrm{K}^{\mp} \pi^{ \pm} \pi^{ \pm}$, selected by requiring secondary displaced vertex with three tracks and a reconstructed invariant mass compatible with the $\mathrm{D}^{ \pm}$mass, $\mathrm{D}^{*+}(2010) \rightarrow \mathrm{D}^{0} \pi^{+}\left(\mathrm{D}^{*-}(2010) \rightarrow \overline{\mathrm{D}}^{0} \pi^{-}\right)$ with subsequent decay $\mathrm{D}^{0} \rightarrow \mathrm{K}^{-} \pi^{+}\left(\overline{\mathrm{D}}^{0} \rightarrow \mathrm{K}^{+} \pi^{-}\right)$, selected by requiring a secondary displaced vertex with two tracks and a reconstructed invariant mass compatible with 
the $\mathrm{D}^{0}$ mass as well as a third track from the primary vertex such that the three track invariant mass is compatible with the $\mathrm{D}^{*+}(2010)$ mass and finally semileptonic decay of the c-quark, selected by requiring a reconstructed muon within the jet.

All three decay channels differentiate between c-quark and anti-quark production, thereby allowing to separate events where the electric charge of the c-jet and the Wboson candidate have the same sign, SS, and where the charges have opposite sign, OS. For most background processes there is no correlation between the charge assigned to the jet and the W-boson, while the signal is purely OS. Therefore, by subtracting corresponding SS distribution form the OS distribution, an almost pure signal can be obtained, with only a small contamination from Drell-Yan remaining.

More details on the event selection and the backgrounds can be found in the original paper [11].

\subsubsection{Measurement}

The number of OS-SS events for each channel is corrected for the branching ratio of the c-quark decay, for the reconstruction (and trigger) efficiency and acceptance and for the integrated luminosity of the data sample, to obtain the total cross-section within the considered fiducial volume, given by the lepton and jet $p_{\mathrm{T}}$ and $\eta$ requirements. The measured total $W+c$ cross-section in this volume is $84.1 \pm 2.0$ (stat) \pm 4.9 (stat). A full description of the systematic uncertainties can be found in the original paper [11]. The largest uncertainties are due to the uncertainty on the c-quark decay branching ratios and on the integrated luminosity.

The measured cross-section is compared with theoretical predictions in figure 6 . The theoretical predictions are at NLO calculated from MсFM [30] and combined with NNLO PDF sets, MSTW2008 [2], CT10 [1], NNPDF2.3 [31] and NNPDF2.3 coll [3]. The uncertainties on the theoretical predictions shown in figure 6 only include the PDF uncertainty. An additional uncertainty of approximately $\pm 5 \%$ is associated with scale variations. The large differences in the size of the PDF uncertainties in figure 6 are due to the parametrization of the individual PDF sets with respect to the s-quark PDF, its correlation with other PDFs (such at the $\bar{d}$ sea quark PDF) and the definition of the uncertainty on the strangeness. The normalized differential cross-section with respect to the pseudorapidity of the lepton associated with the $\mathrm{W}$-boson decay is shown in figure 7.

Finally the $\left(W^{+}+\bar{c}\right)$ to $\left(W^{-}+c\right)$ ratio is measured as a function of $|\eta|$ of the lepton from W-boson decay. The result is shown and compared to the theoretical predictions in figure 8. As most experimental systematic uncertainties cancel in the ratio, the remaining uncertainty is almost fully statistical.

The same analysis was also performed with a lower $p_{\mathrm{T}}$ threshold on the isolated lepton of $25 \mathrm{GeV}$, but only in the muon channel. Results of this analysis can be found in Ref. [11].

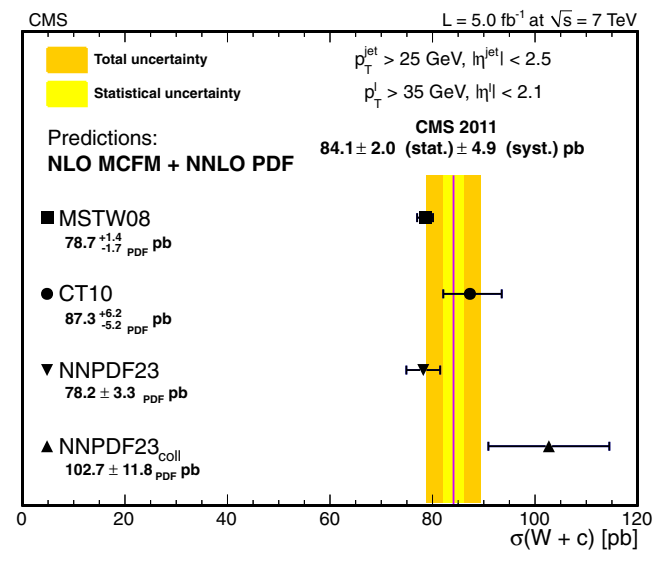

Figure 6. The fiducial cross-section of W-boson production is association with a c-quark for $\mathrm{W}$-bosons decaying to an electron or muon with $p_{\mathrm{T}}>35 \mathrm{GeV}$ and $|\eta|<2.1$ and for c-quarks with $p_{\mathrm{T}}>25 \mathrm{GeV}$ and $|\eta|<2.5$ [11]. The comparison with theoretical NLO predictions using different PDF sets is included.

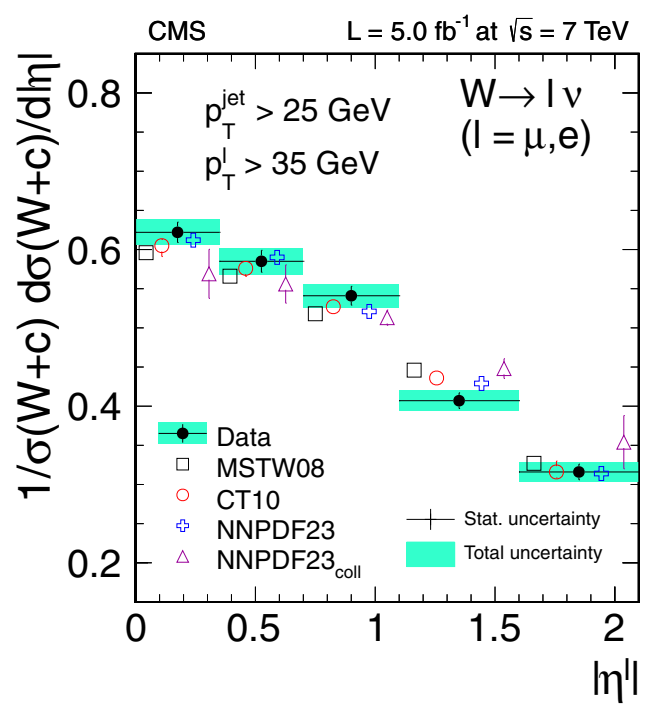

Figure 7. The normalized differential cross-section with respect to $|\eta|$ of the lepton from W-boson decay for W-boson production is association with a c-quark in data (black dots) and from theoretical predictions using different PDF sets (empty markers) [11]. The theoretical predictions are slightly displaced along the horizontal axis for better comparison.

\subsection{Effect on PDFs}

The W-boson muon charge asymmetry measurement described in section 5.1 was included in the HERAFITTER framework. Similarly to the analysis in section 4 a full set of PDFs was fitted both including this CMS measurement and based purely on the HERA-I DIS data. The resulting $\mathrm{u}$ and $\mathrm{d}$ valence quark PDFs are shown in figure 9. A clear reduction in the uncertainty is seen for $x<0.1$.

By adding the $W+c$ measurements from section 5.2 to the fitter, it is possible to separate the s and d sea quark 


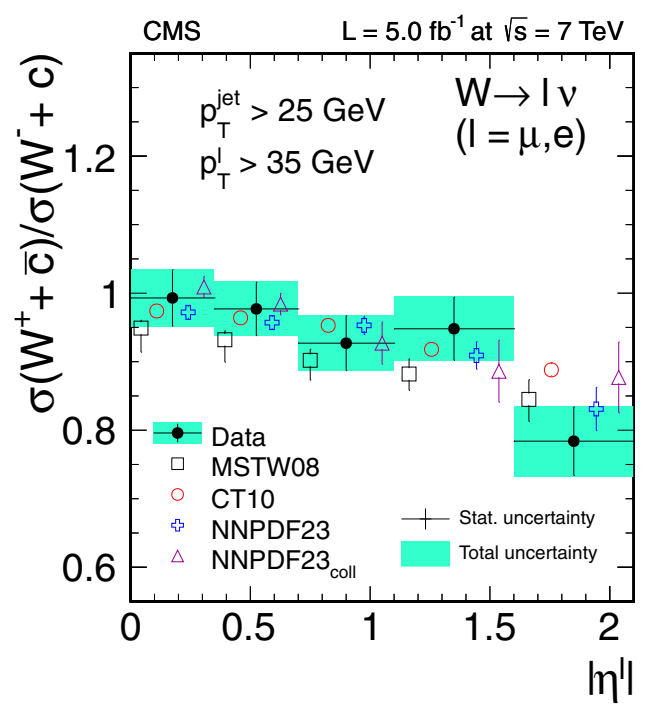

Figure 8. The cross-section ratio $\sigma\left(W^{+}+\bar{c}\right) / \sigma\left(W^{-}+c\right)$ as a function of the pseudo-rapidity of the lepton from the W-boson decay in data (black dots) and from theoretical predictions using different PDF sets (empty markers) [11]. The theoretical predictions are slightly displaced along the horizontal axis for better comparison.

PDFs. This is done by replacing $x \bar{D}(x)$ in equation 1 with

$$
\begin{aligned}
x \bar{d}(x) & =A_{\bar{d}} x^{B_{\bar{d}}}(1-x)^{C_{\bar{d}}} \text { and } \\
x \bar{s}(x) & =A_{\bar{s}} x^{B_{\bar{s}}}(1-x)^{C_{\bar{s}}} .
\end{aligned}
$$

To retain the equal $\bar{u}$ and $\bar{d}$ densities at $x \rightarrow 0, A_{\bar{U}}=A_{\bar{d}}$ and $B_{\bar{U}}=B_{\bar{d}}$ are required. Furthermore $B_{\bar{s}}$ is set to $B_{\bar{d}}$. This leaves 15 free parameters to be fitted.

The fitted s quark fraction $R_{s}\left(x, Q^{2}\right)=(s+\bar{s}) /(\bar{u}+\bar{d})$ is shown in figure 10 . The strange fraction decreases at high $x$ but increases with the energy scale. It approaches 1 for $x<0.001$ for $Q^{2}=m_{W}^{2}$.

\section{Conclusion}

A wide range of CMS measurements are sensitive to PDFs and can be used to improve current PDF sets. As illustrated by the study in section 4 measurements of jet production at the LHC provide a direct probe on the gluon PDFs and can help to significantly reduce their uncertainty compared to DIS data alone. This is especially the case at high $x$ which is the range most relevant for LHC analyses. Jets production, especially in the very forward region also indirectly probes the $\mathrm{u}$ and $\mathrm{d}$ valence quark PDFs.

Gauge bosons at the LHC are predominantly produced in the annihilation of a valence quark with a sea antiquark and therefore their production can be used to limit the quark PDFs. In this paper a W-boson muon charge asymmetry measurement was considered, which significantly reduced the uncertainty on the valence quark PDFs for $x<0.1$. By focusing on gauge-boson production is association with a heavy quark, the heavier quark PDFs can be accessed. The $\mathrm{W}+\mathrm{c}$ production measurements of
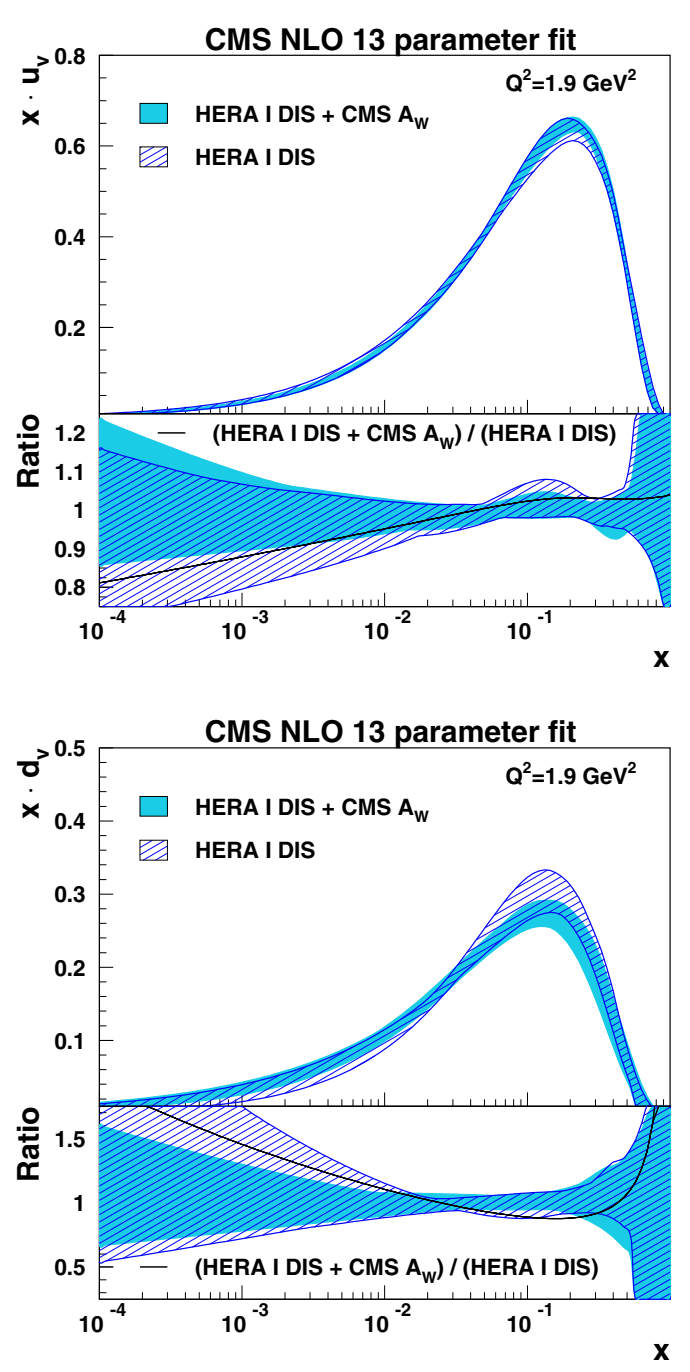

Figure 9. The u (top) and d (bottom) valence quark PDFs fitted on the combination of HERA-I DIS data and the CMS W-boson muon charge asymmetry measurement (filled line) are shown together with the same PDFs fitted without the CMS data (dashed line) [10].

section 5.2 were used to fit an s-quark PDF independent of that of the $\mathrm{d}$ sea quarks.

\section{References}

[1] H.-L. Lai, M. Guzzi, J. Huston, Z. Li, P. M. Nadolsky, J. Pumplin, and C.-P. Yuan, Phys. Rev. D 82, 074024 (2010)

[2] A. D. Martin, W. J. Stirling, R. S. Thorne, and G. Watt, Eur. Phys. J. C 63, 189 (2009)

[3] R. D. Ball et al. Nucl. Phys. B 867, 244 (2013)

[4] H1 and ZEUS Collaboration, J. High Energy Phys. 01, 109 (2010)

[5] S. Alekhin, J. Blümlein, S. Klein, and S. Moch, Phys. Rev. D 81, 014032 (2010)

[6] H1 Collaboration, Eur. Phys. J. C 64, 561 (2009)

[7] HERAFitter web site, http://www.herafitter.org 


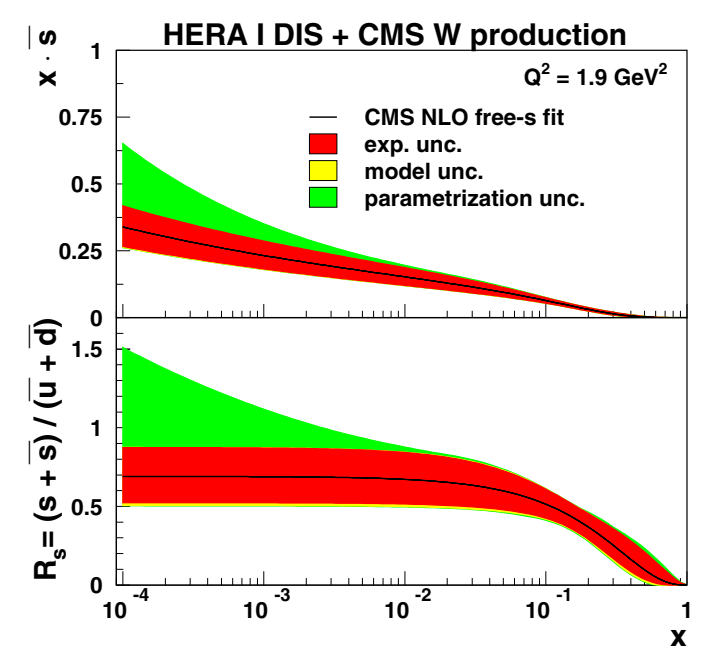

Figure 10. The strange ratio, $R_{s}\left(x, Q^{2}\right)=(s+\bar{s}) /(\bar{u}+\bar{d})$, from PDFs fitted from on the combination of HERA-I DIS data, the CMS W-boson muon charge asymmetry measurement and CMS data from $\mathrm{W}$-boson production in association with a c-(anti)quark [10].

[8] CMS collaboration, Phys. Rev. D 87, 112002 (2013)

[9] CMS collaboration, arXiv:1410.6765 (2014)

[10] CMS collaboration, Phys. Rev. D 90, 032004 (2014)

[11] CMS collaboration, JHEP 02, 013 (2014)

[12] M. Botje, Comput. Phys. Commun 182, 490 (2011)

[13] NuTeV Collaboration, Phys. Rev. Lett. 99, 192001 (2007)

[14] R. S. Thorne, Phys. Rev. D 73054019 (2006)

[15] CMS Collaboration, JINST 03, S08004 (2008)
[16] M. Cacciari, G. P. Salam, and G. Soyez, J. High Energy Phys. 04, 063 (2008)

[17] M. Cacciari and G. P. Salam, Phys. Lett. B 641, 57 (2006)

[18] Z. Nagy, Phys. Rev. D 68, 094002 (2003)

[19] T. Kluge, K. Rabbertz, and M. Wobisch, arXiv:hepph/0609285v2 (2006)

[20] R. D. Ball, L. Debbio, S. Forte, A. Guffanti, J. I. Latorre, J. Rojo, and M. Ubiali, Nucl. Phys. B 838, 136 (2010)

[21] T. Sjöstrand, S. Mrenna, and P. Skands, JHEP 05, 026 (2006)

[22] Bähr et al., Eur. Phys. J. C 58, 639 (2008)

[23] P. Nason, JHEP 11, 040 (2004)

[24] S. Frixione, P. Nason, and C. Oleari, JHEP 11, 070 (2007)

[25] S. Alioli, P. Nason, C. Oleari, and E. Re, JHEP 06, 043 (2010)

[26] S. Alioli, K. Hamilton, P. Nason, C. Oleari and E. Re, JHEP 04, 081 (2011)

[27] CMS Collaboration, CMS Physics Analysis Summary, CMS-PAS-PFT-09-001 (2009)

[28] CMS Collaboration, CMS Physics Analysis Summary, CMS-PAS-PFT-10-001 (2010)

[29] Y. Li and F. Petriello, Phys. Rev. D 86, 094034 (2012)

[30] J. Alwall, M. Herquet, F. Maltoni, O. Mattelaer and T. Stelzer, JHEP 06, 128 (2011)

[31] NNPDF collaboration, R.D. Ball et al. Nucl. Phys. B 855, 153 (2012) 153

[32] A. D. Martin, A.J.Th.M. Mathijssen, W.J. Stirling, R.S. Thorne, B.J.A. Watt and G. Watt, Eur. Phys. J. C 73, 2318 (2013) 